\section{PWE-158 SCREENING IMMIGRANTS ORIGINATING FROM HIGH PREVALENCE AREAS FOR HEPATITIS B USING PRIMARY CARE DATABASES - EXPERIENCE FROM A SINGLE GP PRACTICE WITH A LARGE MIGRANT POPULATION}

${ }^{1}$ VJ Appleby*, ${ }^{2} \mathrm{~A}$ Khan, ${ }^{2} \mathrm{~J}$ Rehman, ${ }^{3} \mathrm{GR}$ Foster, ${ }^{1} \mathrm{~S}$ Moreea. ${ }^{1}$ Gastroenterology, Digestive Diseases Centre, Bradford Royal Infirmary, Duckworth Lane, UK; ${ }^{2}$ Barkerend Health Centre, Bradford, UK; ${ }^{3}$ Blizard Institute of Cell Research, London, UK

\subsection{6/gutjnl-2014-307263.418}

Introduction Chronic Hepatitis B (HBV), a major cause of liver disease, is underdiagnosed in the UK with pockets of high prevalence in areas with large immigrant populations. Identifying individuals through a screening programme dedicated to high risk individuals would be beneficial if that target population could be defined.

Our aims were to interrogate a Primary Care database (SystmOne) establishing the best way of identifying individuals suspected of having had a HBV test to exclude them from the target population.

Methods We used four search terms: age (>18), ethnic code, birth place, language spoken. Read codes (RC) were applied to identify individuals previously tested/diagnosed with HBV. RC were identified by using 'HBV' as a broad search term.

Results We identified 4256 individuals aged $\geq 18$. 718 (18\%) were excluded because of lack of demographic data; 3210 (75\%) qualified as the target population. $81 \mathrm{RC}$ pertaining to $\mathrm{HBV}$ were found and generated 413 'hits', only 224 'hits' (54\%) satisfied our criteria. 206 individuals (6.4\%) had HBV serology recorded, 9/ $3210(0.28 \%)$ were coded for chronic HBV, a further $2 \mathrm{HBsAg}$ positive patients $(0.9 \%)$ had no RC. After reviewing individual notes electronically we established the following $8 \mathrm{RC}$ to be the 'most useful': XaIq7, XaFuS, X306n, X306i, 43B4, XaMBL, XaG1R, XaPEy which would have identified the latter.

Conclusion We have identified the 8 most useful RC to help with a potential screening programme. Using all available HBV RC would be too time consuming. This study highlights that very few high risk immigrants are currently tested for HBV. Disclosure of Interest None Declared.

\section{Neurogastroenterology: functional disorders, motility and clinical physiology}

\section{PWE-159 THE COSTS OF IRRITABLE BOWEL SYNDROME (IBS) IN AN INCREASINGLY COST AWARE NHS}

${ }^{1} \mathrm{P}$ Wilson, ${ }^{1} \mathrm{~A}$ Poullis*, ${ }^{2} \mathrm{~J}$ Wilkins, ${ }^{3} \mathrm{M}$ Rance. ${ }^{1}$ St Georges Hospital NHS Trust, London, UK; ${ }^{2}$ Harvey Walsh Ltd, Runcorn, UK; ${ }^{3}$ Almirall UK, Uxbridge, UK

\subsection{6/gutjnl-2014-307263.419}

Introduction The NHS is faced with increasing cost pressures making the efficient use of resources paramount. Both NICE and BSG guidelines state that patients with IBS should be managed in primary care. ${ }^{1,2}$ Despite this up to $50 \%$ of patients with a diagnosis of IBS are referred to secondary care for investigation. ${ }^{3}$ Outpatient visits and endoscopic investigations consume a considerable NHS resource.

Methods Hospital Episode Statistics (HES) data for 2012-13 for all the Care Commissioning Groups (CCGs) in England were analysed to calculate the financial cost of IBS. IBS symptom codes were included. Organic gastrointestinal (GI) disease codes were excluded from the analysis. Primary Care prescribing analyses and cost (PACT) data 2012-13 were analysed to calculate IBS treatment costs.
Results In England in 2012/13, there were 1,219,961 patients attending gastroenterology and colorectal surgery outpatient specialties, with a total cost of $£ 365,868,937$. Despite this, only a total of 1,982 patients who were coded as IBS with a total estimated cost of $£ 744,812$ were recorded. However, if we look at the total costs of patients under 50 years of age with excluded diagnosis as described above, we get a total of 28,849 patients with a cost of $£ 11,002,874$. In $2011 / 12$, there were also 323,752 day case and outpatient diagnostic endoscopies with a total cost of $£ 169,676,704$ where no further activity was seen either as an inpatient or outpatient for the 12 months following the diagnostic endoscopy. This represents $49 \%$ of the total diagnostic endoscopies performed in this group of patients. In Primary Care, treatment with laxatives and antispasmodics totalled over 50 million pounds, with $£ 40,219,270$ spent on macrogol and $£ 11,024,948$ spent on mebeverine.

Conclusion Despite being poorly clinically coded it is clear that IBS is a significant costs burden to the NHS. Over half of patients seen for day case and outpatient diagnostic endoscopies have no further clinical activity coded over the subsequent 12 months implying functional symptoms. Better diagnosis and subsequent management of IBS within a Primary Care setting would provide direct savings in the cost of IBS management as well as enabling GI services to target its resources such as colonoscopy services towards other GI conditions more appropriately in areas of greater clinical need.

Work funded by Almirall as a "Service to Medicine". Hospital Episode Statistics Data provided via Harvey Walsh Ltd under commercial reuse licence from the Health and Social Care Information Centre 2014.

\section{REFERENCES}

1 N.I. C. E. IBS in Adults. Clinical Guidelines 61. 2008

2 B.S. G. Guidelines on IBS. 2007

3 B.S. G. Commissioning Report on IBS. 2009

Disclosure of Interest P. Wilson: None Declared, A. Poullis: None Declared, J. Wilkins Employee of: Harvey Walsh Ltd, M. Race Employee of: Almirall

\section{PWE-160 THE JOINT HYPERMOBILITY SYNDROME IS ASSOCIATED WITH FUNCTIONAL DYSPEPSIA AND REFLUX AND IDENTIFIES A SUBGROUP WITH SOMATISATION, CHRONIC PAIN AND WORSE QUALITY OF LIFE}

${ }^{1} \mathrm{~A}$ Fikree ${ }^{*},{ }^{1} \mathrm{R}$ Aktar, ${ }^{1} \mathrm{~A}$ Farmer, ${ }^{2} \mathrm{R}$ Grahame, ${ }^{1} \mathrm{C}$ Knowles, ${ }^{1} \mathrm{Q}$ Aziz. ${ }^{1}$ Neurogastroenterology, Digestive Diseases, QMUL; ${ }^{2}$ Rheumatology, University College Hospital, London, UK

\subsection{6/gutjnl-2014-307263.420}

Introduction The Joint hypermobility syndrome (JHS) is a noninflammatory connective tissue disorder with a prevalence of $20 \%$. It is characterised by joint hypermobility,chronic pain, fibromyalgia (FM) and dysautonomia. Gastrointestinal (GI) symptoms e.g., dyspepsia, reflux, bloating and constipation are present in up to $80 \%$ of affected individuals. Small studies suggest that FGID are common in these patients yet no controlled studies have systematically investigated if JHS is associated with particular GI diagnoses nor explored the effect of JHS on nonGI symptom presentation and quality of life (QOL).

Methods Using a nested case-control double-blind study in secondary care GI clinics, consecutive new referrals (without prior GI diagnosis), aged 18-70, completed validated questionnaires for GI, somatic, psychological and autonomic symptoms and QOL, and were assessed for JHS and FM. They then consulted a 